\title{
OTIMIZAÇÃO DE EXPERIMENTOS COM VARIÁVEIS DE RESPOSTA DESCRITAS POR PERFIS
}

\author{
Flávio Sanson Fogliatto \\ Dep. de Engenharia de Produção e Transportes \\ Universidade Federal do Rio Grande do Sul (UFRGS) \\ Porto Alegre - RS \\ ffogliatto@producao.ufrgs.br
}

Recebido em 02/2008; aceito em 09/2008 após 1 revisão Received February 2008; accepted September 2008 after one revision

\begin{abstract}
Resumo
Em experimentos multi-resposta (MREs) a mesma unidade experimental é avaliada em relação a mais de uma resposta simultaneamente. A otimização de MREs implica na determinação do ponto na superfície de resposta onde as respostas apresentam o melhor desempenho em relação aos critérios estabelecidos. Funções de utilidade são empregadas para transformar realizações das respostas em cada tratamento experimental em medidas de desempenho. Este artigo aborda MREs em que algumas respostas não são observadas como valores individuais, mas descritas por funções. Uma resposta funcional apresenta um ou mais perfis como resultados observados em cada tratamento experimental; o objetivo é identificar tratamentos que resultam em respostas próximas de um perfil-alvo. Neste artigo, propõe-se a utilização da distância de Hausdorff, uma métrica de similaridade utilizada no tratamento de imagens, em combinação com uma função de preferência para obter uma função de utilidade que forneça a distância das respostas funcionais em relação a seu perfil-alvo.
\end{abstract}

Palavras-chave: variáveis funcionais; distância de Hausdorff; otimização multi-resposta.

\begin{abstract}
In multiresponse experiments (MREs) the same experimental unit is evaluated with respect to more than one response simultaneously. Optimization of MREs involves determining the point in the design region where responses perform best with respect to given criteria. Utility functions are used to transform responses outcomes at each experimental treatment into performance measures. In this paper, we investigate MREs where some response outcomes are profiles rather than individual values. A functional response gives one or more profiles as observed outcomes at each experimental treatment, and the objective is to identify the outcome that is closest to a target profile. We propose the use of the Hausdorff Distance, a similarity metric from the field of image recognition, in combination with a desirability function to obtain a utility function that gives the distance of a functional response outcome to its desired target.
\end{abstract}

Keywords: functional variables; Hausdorff distance; multiresponse optimization. 


\section{Introdução}

Em alguns ramos industriais, o desempenho dos itens produzidos é avaliado a partir de múltiplos critérios. Ao desenvolver tais produtos, é necessário otimizar conjuntamente os critérios de forma a obter a melhor configuração de produto, em um procedimento conhecido como Otimização Multi-resposta (OM ou Multiresponse Optimization; Khuri \& Cornell, 1996). Alguns dos critérios considerados podem estar relacionados a respostas quantitativas, mensuradas usando equipamentos laboratoriais ou painéis de avaliação (por exemplo, painéis sensoriais); outros critérios podem envolver a opinião de especialistas (por exemplo, ao se avaliar a importância de uma resposta com relação às demais). No desenvolvimento do produto, o engenheiro de P\&D (Pesquisa e Desenvolvimento) usualmente executa um experimento planejado que resulta em amostras de produtos, as quais são avaliadas relativamente a critérios pré-definidos, na busca da melhor configuração (que pode ser uma dada formulação ou combinação de ajustes para as variáveis de processo, por exemplo) para o produto sendo desenvolvido.

Procedimentos de OM são usualmente operacionalizados em quatro etapas (Fogliatto \& Albin, 2000): (i) as variáveis de resposta são modeladas como funções dos fatores experimentais de controle usando técnicas de regressão; (ii) medidas preditas de desempenho (por exemplo, distância-ao-alvo) são calculadas usando valores preditos das variáveis de resposta; (iii) as medidas de desempenho são combinadas considerando todas as variáveis de resposta através de algum procedimento ou função utilidade; e (iv) a função utilidade é otimizada de forma a identificar os melhores ajustes para os fatores experimentais de controle.

$\mathrm{Na}$ etapa (ii) acima, pressupõe-se que, associado a um dado tratamento experimental nãoreplicado (ou seja, a uma combinação de ajustes dos fatores de controle), existirá um valor único observado para cada variável de resposta considerada no experimento. Entretanto, observações de algumas variáveis de resposta podem não ser plenamente informativas se expressas em termos de um valor individual. Por exemplo, ao avaliar a usabilidade de um adoçante dietético a uma dada temperatura, o perfil de solubilidade do composto (isto é, uma curva descrevendo sua solubilidade em água ao longo do tempo) pode ser mais informativo para o engenheiro de P\&D do que uma medida individual de solubilidade. Analogamente, o conhecimento do perfil de sabor do adoçante pode ser mais importante, durante o desenvolvimento de um produto dietético, do que o seu sabor residual percebido, por exemplo, após 10 segundos da ingestão. Desta forma, ao otimizar certos produtos pode ser mais importante obter uma amostra final com variáveis de resposta apresentando valores próximos a perfisalvo do que a valores-alvo. O estado-da-arte da literatura sobre OM não apresenta estratégias que permitam considerar tais variáveis funcionais de resposta na otimização experimental.

Neste artigo, propõe-se a utilização da distância de Hausdorff (DH) em procedimentos de otimização multi-resposta. A DH foi originalmente proposta por Huttenlocher et al. (1993) para aplicações na área de reconhecimento de imagens. Os autores desenvolveram um método correlacional usando a DH para determinar a existência de padrões-modelo em imagens. A DH fornece uma medida da distância entre pontos em dois perfis, tal que valores pequenos de distância indicam perfis similares entre si. A DH permite que se incorpore a distância a um perfil-alvo como critério de otimização em experimentos multi-resposta, como introduzido na seqüência.

Funções utilidade usadas em OM reescrevem valores observados das variáveis de resposta em diferentes tratamentos experimentais em termos de critérios de desempenho prédefinidos, tais como distância-ao-alvo e sensibilidade a perturbações nos ajustes dos fatores 
de controle. Tais funções somente permitem a utilização de valores individuais como informações de entrada. Entretanto, variáveis de resposta funcionais são caracterizadas por observações dadas por perfis, e não por valores individuais (como no caso de variáveis de resposta ordinárias). Relacionados a variáveis de resposta funcionais existe um ou mais perfis observados a cada tratamento experimental e, no contexto de uma otimização, o objetivo é identificar o perfil mais próximo a um perfil nominal ou alvo. Neste artigo, utilizase a DH para obter uma medida individual que expresse a proximidade de um determinado perfil observado ao perfil-padrão, transformando, assim, variáveis de resposta funcionais em ordinárias. As respostas reescritas através da $\mathrm{DH}$ apresentam valores-alvo iguais a zero e suas observações podem ser convertidas em valores de preferência, utilizando a função proposta por Derringer \& Suich (1980). Valores de preferência são restritos ao intervalo $[0,1]$ sendo, assim, comparáveis mesmo se oriundos de diferentes variáveis de resposta, como demandado na análise de experimentos multi-resposta. As medidas de desempenho associadas às diferentes variáveis de resposta são finalmente combinadas usando a média geométrica dos valores preditos de preferência.

Para ilustrar o uso da DH na otimização de experimentos multi-resposta, estende-se o estudo de caso originalmente apresentado em Fogliatto \& Albin (2003), no qual discute-se a otimização de um produto para animais. No estudo em questão, variáveis de resposta avaliadas através de painéis sensoriais devem ser otimizadas em conjunto com outras variáveis, mensuradas utilizando equipamentos de laboratório. A textura do produto é uma das variáveis de resposta sensoriais consideradas no experimento. Neste artigo, essa variável é substituída por uma resposta funcional, obtida simulando testes de compressão no produto (um biscoito para cães).

O presente trabalho está diretamente relacionado a pesquisas sobre a análise de experimentos multi-resposta. Diversos autores propuseram diferentes funções para combinar as múltiplas respostas. Um sumário de referências relevantes vem dado na Figura 1, agrupadas, segundo seu referencial teórico, em cinco abordagens: (i) relacionadas à metodologia de superfície de resposta; (ii) baseadas na função de preferência; (iii) baseadas na teoria de projeto robusto de Taguchi; (iv) relacionadas a redes neurais e lógica difusa; e (v) relacionadas a índices de capacidade de processo. As abordagens (ii) e (iii) são claramente as mais investigadas na literatura sobre $\mathrm{OM}$, como demonstra o número de referências associadas a elas. Nenhum dos trabalhos listados na Figura 1 traz abordagens capazes de lidar com características de qualidade funcionais, o que motivou os desenvolvimentos apresentados neste artigo.

Algumas referências adicionais àquelas na Figura 1 merecem menção. Murphy et al. (2005) revisaram a literatura sobre otimização de experimentos uni-resposta e multi-resposta, cobrindo parte dos trabalhos listados na Figura 1. Wurl \& Albin (1999) e Kros \& Mastrangelo (2001) compararam funções, propostas na literatura sobre $\mathrm{OM}$, para combinar variáveis de resposta. Esses trabalhos trazem uma análise aprofundada da função de preferência e suas variantes bem como da função de perda de Taguchi (1986) e suas variantes.

O número de publicações sobre a análise de variáveis funcionais cresceu significativamente nos últimos anos, conforme reportado nas obras de Ramsay \& Silverman (2005) e Ferraty \& Vieu (2006). Técnicas e ferramentas estatísticas consagradas têm sido adaptadas para permitir a análise de dados funcionais, com destaque para os métodos de análise multivariada, conforme reportado por Manteiga \& Vieu (2007). Trabalhos relacionados à área de Engenharia da Qualidade abordam estratégias para modelar variáveis de resposta funcionais (e.g., Cardot et al., 1999; Nair et al., 2002; Govaerts \& Noël, 2005; Shen \& Xu, 2007) e para permitir o controle estatístico da qualidade de processos ou produtos descritos 
por variáveis funcionais (e.g., Kang \& Albin, 2000; Kim et al., 2003; Jeong et al., 2006; Williams et al., 2007). Até a finalização deste artigo, não havia sido encontrados trabalhos abordando a otimização de experimentos multi-resposta incluindo variáveis funcionais.

$\mathrm{O}$ restante deste artigo está assim organizado. O critério de otimização distância-ao-perfilalvo aqui proposto e sua utilização em conjunto com a função de preferência estão apresentados na seção 2 . A seção 3 traz um estudo de caso onde a utilização do novo critério de otimização é ilustrada. Uma conclusão encerra o artigo na seção 4.

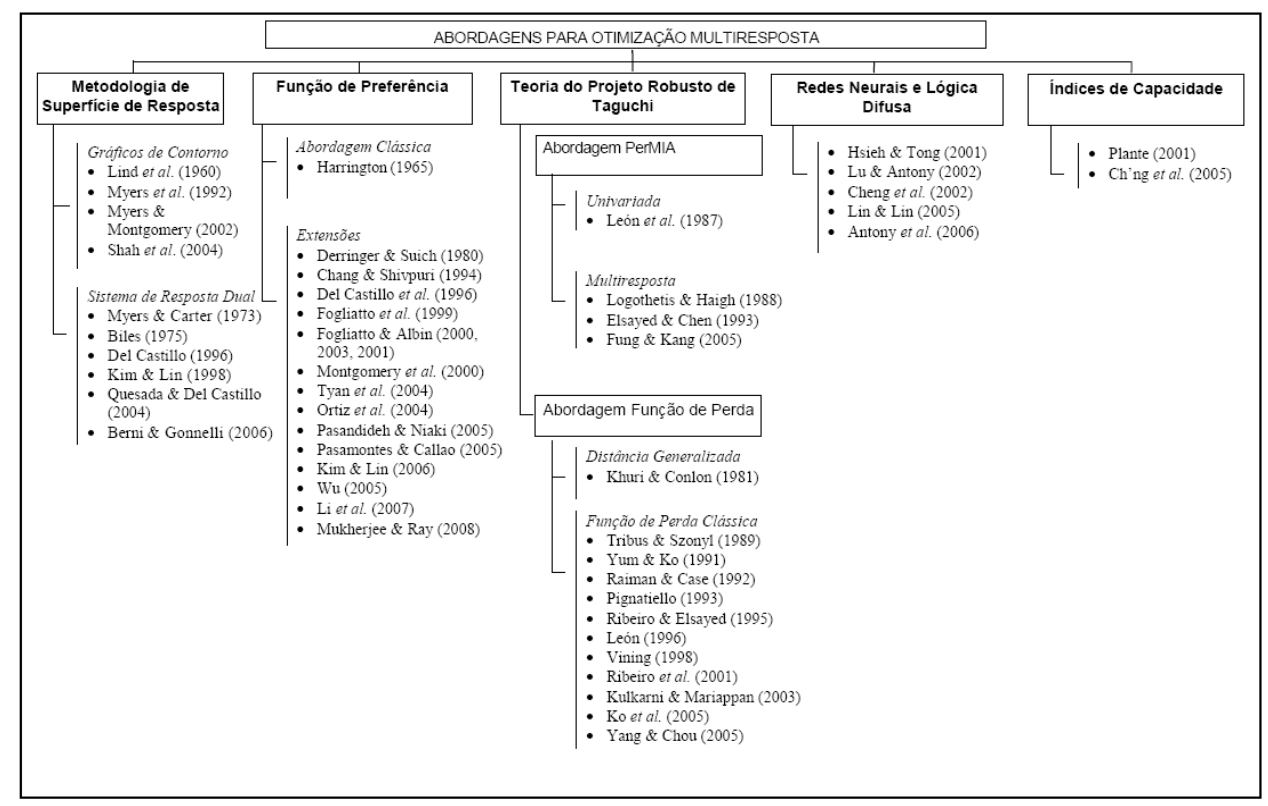

Figura 1 - Abordagens para otimização multi-resposta na literatura.

\section{Método}

A distância de Hausdorff (DH) fornece uma medida da distância entre pontos em dois conjuntos de dados (por exemplo, perfis), tal que valores pequenos de distância indicam conjuntos similares entre si. Propõe-se aqui a utilização da DH para comparar observações de variáveis de resposta funcionais com um perfil nominal ou alvo e, em combinação com a função de preferência, obter uma função de utilidade que forneça a distância entre a observação da variável de resposta funcional e seu alvo desejado.

A DH pode ser assim definida (Huttenlocher \& Kedem, 1990; Huttenlocher et al., 1993; Belogay et al., 1997). Sejam $\mathbf{x}$ e $\mathbf{y}$ dois vetores tais que:

$$
\mathbf{x}^{t}=\left(\begin{array}{llll}
x_{1} & x_{2} & \cdots & x_{m}
\end{array}\right) \quad \mathbf{y}^{t}=\left(\begin{array}{llll}
y_{1} & y_{2} & \cdots & y_{n}
\end{array}\right),
$$

onde $m$ e $n$ não são necessariamente idênticos. A distância de Hausdorff entre os vetores $\mathbf{x} \mathrm{e}$ $\mathbf{y}$ é designada por $d_{\mathbf{y}}(\mathbf{x})$ e determinada como:

$$
d_{\mathbf{y}}(\mathbf{x})=\max _{x \in \mathbf{x}} d(x, \mathbf{y}) .
$$


A distância $d(x, \mathbf{y})$ entre a observação $x$ e o vetor $\mathbf{y}$ é dada por:

$$
d(x, \mathbf{y})=\min _{y \in \mathbf{y}} d(x, y)
$$

onde $d(x, y)$ é uma métrica qualquer de distância entre pontos $x$ e $y$ em $\mathbf{x}$ e $\mathbf{y}$, respectivamente. Em outras palavras, a distância $d(x, \mathbf{y})$ na eq. (3) é dada pela mínima distância entre uma dada observação $x$ pertencente ao vetor $\mathbf{x}$ e todas as observações $y$ pertencentes ao vetor $\mathbf{y}$, consideradas uma a uma. No total, $n$ distâncias serão comparadas, das quais se tomará a de menor valor.

A métrica utilizada em $d(x, y)$ pode ser definida, por exemplo, como a distância Euclidiana entre um par de pontos $x$ e $y$; isto é:

$$
e(x, y)=\sqrt{(x-y)^{2}} \text {. }
$$

Funções maximin, como aquela apresentada na eq. (2), são assimétricas, ou seja, $d_{\mathbf{y}}(\mathbf{x})$ e $d_{\mathbf{x}}(\mathbf{y})$ não necessariamente fornecem o mesmo resultado. Uma definição mais geral da distância de Hausdorff, usada no restante deste artigo, leva em consideração essa assimetria, sendo dada por:

$$
h(\mathbf{x}, \mathbf{y})=\max \left\{d_{\mathbf{y}}(\mathbf{x}), d_{\mathbf{x}}(\mathbf{y})\right\} .
$$

A eq. (5) fornece uma interpretação geométrica intuitiva da DH como o limite superior da mínima distância entre pontos (ou vértices) nos vetores $\mathbf{x}$ e $\mathbf{y}$. Parece claro que tal limite pode ser afetado pela existência de valores extremos nas distâncias mínimas, o que pode não ser desejável. Para contornar esse problema, alguns autores propuseram substituir o operador de máximo na eq. (2) pela mediana (Castagliola \& Rosa, 2006) ou pela soma (Belogay et al., 1997) das distâncias $d_{\mathbf{y}}(\mathbf{x})$ e $d_{\mathbf{x}}(\mathbf{y})$. É importante destacar que os desenvolvimentos associados à DH apresentados para dois vetores podem ser generalizados para conjuntos de dados de maior dimensão. Por fim, quando a eq. (4) for a métrica selecionada para utilização na eq. (3), a distância de Hausdorff resultante na eq. (5) é designada por $h^{E}(\mathbf{x}, \mathbf{y})$.

Para ilustrar a utilização das equações (1) a (4), bem como da eq. (5) e seus possíveis operadores (máximo, média, mediana e soma), considere os vetores de dados correspondentes aos tratamentos $t=0$, aqui designado por $\mathbf{x}$ e composto por 46 observações (isto é, $m=46$ ), e $t=1$, aqui designado por $\mathbf{y}$ e composto por 31 observações (isto é, $n=31$ ). Aplicando as equações (3) e (4) aos dados do exemplo, obtêm-se os 46 valores de $d(x, \mathbf{y})$ e 31 valores de $d(\mathbf{y}, x)$ dados na Tabela 1. Aplicando a eq. (2) ao par de vetores $\mathbf{x}$ e $\mathbf{y}$, obtêm-se $d_{\mathbf{y}}(\mathbf{x})=\max _{x \in \mathbf{x}}\{d(x, \mathbf{y})\}=0,125$ e $d_{\mathbf{x}}(\mathbf{y})=\max _{y \in \mathbf{y}}\{d(y, \mathbf{x})\}=0,152$, ou seja, $d_{\mathbf{y}}(\mathbf{x}) \neq d_{\mathbf{x}}(\mathbf{y})$. Conseqüentemente, o resultado da aplicação da eq. (5) aos dados do exemplo é $h^{E}(\mathbf{x}, \mathbf{y})=0,152$. Substituindo o operador de máximo na eq. (2) pelos operadores de média, mediana e soma, a eq. (5) gera os resultados $h_{\text {avg }}^{E}(\mathbf{x}, \mathbf{y})=0,0367, h_{\text {med }}^{E}(\mathbf{x}, \mathbf{y})=0,0329 \mathrm{e}$ $h_{\text {sum }}^{E}(\mathbf{x}, \mathbf{y})=2,901$, respectivamente, os quais podem ser verificados utilizando os valores na Tabela 1.

Pesquisa Operacional, v.28, n.3, p.577-595, Setembro a Dezembro de 2008 
Tabela 1 - Operacionalização das eqs. (1) a (5) através de um exemplo.

\begin{tabular}{|c|c|}
\hline$d(x, \mathbf{y})$ & $\begin{array}{l}0 ; 0,004 ; 0,125 ; 0,044 ; 0,027 ; 0 ; 0,007 ; 0,053 ; 0,004 ; 0,038 ; 0,047 ; 0,109 ; 0,063 ; \\
0,004 ; 0,013 ; 0,085 ; 0,021 ; 0,009 ; 0,047 ; 0,072 ; 0,117 ; 0,035 ; 0,033 ; 0,075 ; 0,003 ; \\
0,118 ; 0,061 ; 0,044 ; 0,074 ; 0,041 ; 0,031 ; 0,004 ; 0,037 ; 0,067 ; 0,051 ; 0,035 ; 0,024 ; \\
0,016 ; 0,01 ; 0,007 ; 0,004 ; 0,003 ; 0,002 ; 0,001 ; 0 ; 0\end{array}$ \\
\hline$d(\mathbf{y}, x)$ & $\begin{array}{l}0 ; 0,004 ; 0,125 ; 0,075 ; 0,027 ; 0,043 ; 0,113 ; 0,037 ; 0,004 ; 0,047 ; 0,072 ; 0,004 ; \\
0,056 ; 0,036 ; 0,021 ; 0,011 ; 0,031 ; 0,038 ; 0,047 ; 0,007 ; 0 ; 0,152 ; 0,097 ; 0,033 ; \\
0,044 ; 0,003 ; 0,061 ; 0,044 ; 0,004 ; 0 ; 0\end{array}$ \\
\hline
\end{tabular}

A utilização da DH como medida de distância entre pares de conjuntos de pontos pode ser justificada de três maneiras. Primeiro, a utilização da eq. (2) não demanda vetores $\mathbf{x}$ e $\mathbf{y}$ de mesma dimensão. Isso não ocorre, por exemplo, quando a distância Euclidiana entre vetores $\mathbf{x}$ e $\mathbf{y}$, dada por:

$$
e(\mathbf{x}, \mathbf{y})=\left(\sum_{i=1}^{m}\left(x_{i}-y_{i}\right)^{2}\right)^{0,5}
$$

for utilizada. Na eq. (6), os vetores $\mathbf{x}$ e $\mathbf{y}$ devem apresentar o mesmo número de elementos ou ser modificados tal que isso se verifique (via deflexão temporal, por exemplo). Segundo, o cálculo da $\mathrm{DH}$ pode ser realizado utilizando conjuntos de dados sem tratamento prévio, isto é, não é necessário ajustar os perfis a funções matemáticas específicas. Terceiro, se comparada à distância Euclidiana, a DH tende a fornecer uma medida mais realista da distância entre dois vetores de dados, em particular quando o operador de máximo na eq. (2) for substituído por um operador de mediana ou média, conforme ilustrado na seqüência.

Considere, por exemplo, os vetores $\quad \mathbf{x}^{t}=(2,5 ; 11,5 ; 31,5 ; 33,0 ; 53,0 ; 61,0) \quad$ e $\mathbf{y}^{t}=(6,5 ; 30,0 ; 39,0 ; 49,0 ; 68,5 ; 72,0)$, grafados na Figura 2 , e cujas observações são ordenadas no tempo. Os perfis associados aos vetores são similares; entretanto, as medidas de distância fornecem resultados diferentes. Quando a distância Euclidiana é calculada usando a eq. (6), o resultado é $e(\mathbf{x}, \mathbf{y})=32,12$; a $\mathrm{DH}$, obtida através das eqs. (2) a (5), é $h^{E}(\mathbf{x}, \mathbf{y})=11,00 ;$ a DH, obtida usando as eqs. (2) a (5) e substituindo o operador de máximo na eq. (2) por um operador de média, resulta em $h_{a v g}^{E}(\mathbf{x}, \mathbf{y})=4,92$. O pareamento dos valores ordenados nos vetores $\mathbf{x}$ e $\mathbf{y}$, necessário para calcular $e(\mathbf{x}, \mathbf{y})$, é responsável pelo alto valor resultante, ainda que os perfis na Figura 2 sejam similares, apesar de levemente desalinhados. Analisando as eqs. (2) e (3), conclui-se que tal distorção não ocorre quando a DH é utilizada. 


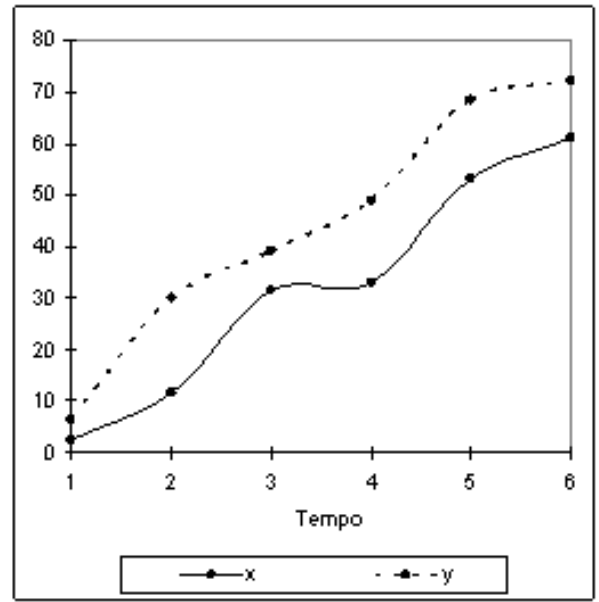

Figura 2 - Perfis correspondentes aos vetores de dados $\mathbf{x}$ e $\mathbf{y}$.

Para um par idêntico de vetores $\mathbf{x}$ e $\mathbf{y}, h^{E}(\mathbf{x}, \mathbf{y})=0$, o que estabelece um valor-alvo para $h^{E}(\mathbf{x}, \mathbf{y})$, bastando, para tanto, que um dos vetores seja definido como contendo o perfil nominal. O critério de otimização distância-ao-perfil-alvo é obtido introduzindo o resultado obtido na eq. (5) na função de preferência de Derringer \& Suich (1980), apresentada na seqüência.

Para medir a proximidade entre uma resposta observada e seu valor-alvo, Derringer \& Suich (1980) propuseram a função de preferência, que converte observações de uma variável de resposta em valores no intervalo [0,1], onde 1 é o valor mais desejável e 0 é inaceitável. Seja $d_{p t}$ o valor de preferência associado à observação $y_{p t}$, obtida a partir da $p$-ésima variável de resposta $(p=1, \ldots, P)$ no $t$-ésimo tratamento experimental $(t=1, \ldots, T)$; isto é,

$$
d_{p t}=\left(\frac{y_{p t}-L_{p}}{\tau_{p}-L_{p}}\right) \cdot I_{\left[L_{p}, \tau_{p}\right]}\left(y_{p t}\right)+\left(\frac{y_{p t}-U_{p}}{\tau_{p}-U_{p}}\right) \cdot I_{\left(\tau_{p}, U_{p}\right]}\left(y_{p t}\right),
$$

onde $\tau_{p}$ é o valor-alvo, e as constantes $L_{p}$ e $U_{p}$ os limites inferior e superior de especificação, respectivamente, associados à $p$-ésima variável de resposta; $I_{[a, b)}$ é uma função indicadora tal que:

$$
I_{[a, b)}=\left\{\begin{array}{l}
1, \text { se } a \leq y_{p t}<b \\
0, \text { caso contrário }
\end{array} .\right.
$$

A eq. (7) é aplicada para o cálculo dos valores de preferência de respostas com alvos dados por números reais diferentes de zero. Caso a variável de resposta apresente somente um limite superior de especificação, a expressão em (7) é reescrita como:

$$
d_{p t}=\left(\frac{y_{p t}-U_{p}}{L_{p}-U_{p}}\right) \cdot I_{\left[L_{p}, U_{p}\right]}\left(y_{p t}\right)+I_{\left(0, L_{p}\right]}\left(y_{p t}\right),
$$


onde $L_{p}$ representa um valor limítrofe, a partir do qual valores inferiores observados para a resposta $p$ são igualmente desejáveis e atribuídos um valor de preferência igual a $1 ; U_{p}$ permanece como definido na eq. (7). Finalmente, para casos onde a resposta apresentar somente um limite inferior de especificação, a expressão em (7) é reescrita como:

$$
d_{p t}=\left(\frac{y_{p t}-L_{p}}{U_{p}-L_{p}}\right) \cdot I_{\left[L_{p}, U_{p}\right]}\left(y_{p t}\right)+I_{\left(U_{p}, \infty\right]}\left(y_{p t}\right),
$$

onde $U_{p}$ corresponde ao valor limítrofe a partir do qual valores superiores observados para a resposta $p$ são igualmente desejáveis e atribuídos um valor de preferência igual a $1 ; L_{p}$ permanece como definido na eq. (7).

Considere uma resposta funcional $Y$ e seus vetores de observações $\mathbf{y}$, obtidos nos diferentes tratamentos experimentais, os quais podem ou não ser replicados. As funções de preferência nas eqs. (7), (9) e (10) não foram desenvolvidas de forma a aceitar observações das variáveis de resposta na forma de vetores como informações de entrada. Assim, para calcular valores de preferência para uma resposta funcional $Y$ é necessário, inicialmente, converter seus vetores observados $\mathbf{y}$, os quais são conjuntos de pontos, em valores observados individuais $y$, sendo cada valor associado a um tratamento experimental não-replicado. Para tanto, propõese substituir as observações funcionais $\mathbf{y}$ pelas medidas de distância-ao-perfil-alvo, dadas pela distância de Haussdorf.

Seja $\mathbf{t}$ o perfil-alvo, e $\mathbf{y}_{p t}$ o vetor observado da variável de resposta funcional $Y_{p}$ no tratamento experimental $t$. Utilizando as eqs. (1) a (5), obtém-se $h_{p t}^{E}$, i.e., o valor de distância de Hausdorff a ser substituído pelo vetor $\mathbf{y}_{p t}$ e usado no restante deste procedimento. Na eq. (1), seja $\mathbf{x}^{t}=\mathbf{t}^{t}$ e $\mathbf{y}^{t}=\mathbf{y}_{p t}^{t}$; alternativamente, o operador de mediana pode ser usado na eq. (2). Seja $\min _{\text {todo } t}\left(h_{p t}^{E}\right)=L_{p}$ e $\max _{\text {todo } t}\left(h_{p t}^{E}\right)=U_{p}$. Inserindo-se as distâncias $h_{p t}^{E}$ para $t=1, \ldots, T$ na eq. (9), obtêm-se as medidas de distância-ao-perfil-alvo associados à resposta $Y_{p}$. Esses valores serão usados, em conjunto com as medidas de preferência obtidas para as demais respostas, em um procedimento de otimização multi-resposta para determinar os ajustes dos fatores de controle que otimizam o experimento multi-resposta. Para tanto, Derringer \& Suich (1980) propõem utilizar a média geométrica para obter uma medida global de preferência para as variáveis de resposta combinadas; isto é,

$$
D_{t}=\left(\prod_{p=1}^{P} d_{p t}\right)^{1 / p}, t=1, \ldots, T .
$$

$\mathrm{Na}$ eq. (11), todas as respostas são igualmente ponderadas em importância. Quando este não for o caso, $\mathrm{Wu}$ (2005) propõe a seguinte extensão da expressão de preferência combinada, de forma a refletir as diferenças de importância entre as variáveis de resposta. Seja $w_{p}$ o peso de importância da $p$-ésima resposta, tal que $0<w_{p}<1$ e $\sum_{p=1}^{P} w_{p}=1$. Uma função de preferência combinada ponderada será dada por: 


$$
D_{t}=d_{1 t}^{w_{1}} \times \ldots \times d_{p t}^{w_{p}}
$$

onde $d_{p t}^{w_{p}}$ designa o valor de preferência ponderado da $p$-ésima variável de resposta $(p=1, \ldots, P)$ no $t$-ésimo tratamento experimental $(t=1, \ldots, T)$, onde $w_{p}$ é o elemento ponderador. Observe que a eq. (12) é uma extensão da expressão clássica para cálculo da média geométrica ponderada proposta por Siegel (1942), para casos onde a soma dos pesos é unitária.

\section{Estudo de caso}

Fogliatto \& Albin (2003) apresentam um estudo de caso reportando o desenvolvimento de uma formulação alternativa para uma marca conhecida de alimento para animais (um biscoito para cães). Dez amostras do produto foram obtidas de um experimento com misturas, onde o percentual de três ingredientes e a espessura do biscoito foram os fatores experimentais de controle. Os níveis de cada ingrediente foram definidos de acordo com um Projeto Composto Central (CCD - Central Composite Design) desenhado para duas variáveis independentes. [As variáveis de mistura foram re-escritas como variáveis independentes utilizando o procedimento proposto por Cornell (1990; p.111-115 e 136-138). Tal procedimento permite $(i)$ o uso de projetos experimentais tradicionais ao invés de projetos experimentais simplex para misturas, e (ii) a utilização de ANOVA e técnicas de regressão na análise dos resultados, o que não é possível no caso de projetos simplex; como ponto negativo, o uso de variáveis independentes tende a restringir a investigação da região experimental.]

A matriz de projeto utilizada, com as variáveis independentes de mistura designadas por $w_{1} \mathrm{e}$ $w_{2}$, bem como as proporções dos ingredientes originais testadas em cada rodada experimental, vêm dadas na Tabela $2(70,3 \%$ da composição da formulação foi investigada no experimento). A espessura do biscoito, designada por $z$ e também apresentada na Tabela 2, foi a única variável de processo incluída no experimento; seus dois níveis correspondem a duas pressões operacionais distintas testadas na extrusora. $\mathrm{O}$ fator $z$ foi usado para dividir o CCD em dois blocos com 5 tratamentos experimentais cada, rodados em turnos consecutivos de um mesmo dia.

Doze variáveis de resposta foram consideradas no experimento original: nove foram medidas usando equipamento laboratorial e três usando painéis sensoriais. Foram selecionadas três respostas laboratoriais com diferentes tipos de alvo para realizar a presente análise. São elas: $Y_{1}=\%$ Umidade, $Y_{2}=$ Espessura $(\mathrm{mm})$, e $Y_{3}=$ Densidade $(\mathrm{g} / \mathrm{ml})$, com valores observados apresentados na Tabela 2 (todas as respostas foram mensuradas no produto final seco). As especificações e tipos de variáveis de resposta são os seguintes: $Y_{1}=$ nominal-é-melhor $\left(U_{1}=8,0 ; \tau_{1}=7,5 ; L_{1}=5,0\right), Y_{2}=$ nominal-é-melhor $\left(U_{2}=10,0 ; \tau_{2}=9,5 ; L_{2}=8,0\right)$, $Y_{3}=$ maior-é-melhor $\left(U_{3}=0,8 ; L_{3}=0,6\right)$. 
Fogliatto - Otimização de experimentos com variáveis de resposta descritas por perfis

Tabela 2 - Rodadas experimentais em termos dos ingredientes da mistura $(x)$ e das variáveis independentes codificadas ( $w$ e $z$ ), observações e valores de preferência das três respostas.

\begin{tabular}{|c|c|c|c|c|c|c|c|c|c|c|}
\hline Trt $(\boldsymbol{t})$ & $\mathbf{1}$ & $\mathbf{2}$ & $\mathbf{3}$ & $\mathbf{4}$ & $\mathbf{5}$ & $\mathbf{6}$ & $\mathbf{7}$ & $\mathbf{8}$ & $\mathbf{9}$ & $\mathbf{1 0}$ \\
\hline \hline$x_{1}(\%)$ & 9,83 & 9,48 & 4,32 & 3,96 & 6,9 & 10,79 & 3,01 & 7,15 & 6,65 & 6,9 \\
\hline$x_{2}(\%)$ & 4,32 & 3,96 & 9,83 & 9,48 & 6,9 & 3,01 & 10,79 & 7,15 & 6,65 & 6,9 \\
\hline$x_{3}(\%)$ & 56,15 & 56,86 & 56,15 & 56,86 & 56,5 & 56,5 & 56,5 & 56 & 57 & 56,5 \\
\hline$w_{1}$ & -1 & -1 & 1 & 1 & 0 & $-1,41$ & 1,41 & 0 & 0 & 0 \\
\hline$w_{2}$ & -1 & 1 & -1 & 1 & 0 & 0 & 0 & $-1,41$ & 1,41 & 0 \\
\hline$z$ & -1 & -1 & -1 & -1 & -1 & 1 & 1 & 1 & 1 & 1 \\
\hline$Y_{1 t}$ & 5,1 & 5,4 & 6,1 & 6,2 & 5,6 & 5,8 & 7,3 & 7,6 & 7,9 & 5,1 \\
\hline$Y_{2 t}$ & 8,30 & 8,24 & 8,49 & 8,48 & 8,17 & 9,0 & 9,56 & 9,28 & 9,62 & 8,95 \\
\hline$Y_{3 t}$ & 0,78 & 0,72 & 0,70 & 0,77 & 0,78 & 0,65 & 0,67 & 0,69 & 0,65 & 0,62 \\
\hline$d_{1 t}$ & 0,040 & 0,160 & 0,440 & 0,480 & 0,240 & 0,320 & 0,920 & 0,800 & 0,199 & 0,039 \\
\hline$d_{2 t}$ & 0,200 & 0,160 & 0,327 & 0,320 & 0,113 & 0,667 & 0,879 & 0,853 & 0,760 & 0,633 \\
\hline$d_{3 t}$ & 0,900 & 0,600 & 0,500 & 0,850 & 0,900 & 0,250 & 0,350 & 0,450 & 0,250 & 0,100 \\
\hline
\end{tabular}

Modelos de regressão linear múltipla, relacionando respostas a fatores experimentais de controle, foram desenvolvidos para $Y_{1}, Y_{2}$ e $Y_{3}$. Para tanto utilizou-se uma implementação computacional do método dos mínimos quadrados. Tais modelos são apresentados nas eqs. (13) a (15); os números entre parênteses são valores $p$ de significância associados aos termos dos modelos. Valores de preferência associados a cada valor predito das respostas nos tratamentos $t=1, \ldots, 10$, foram calculados usando as eqs. (7) e (10), sendo dados na Tabela 2.

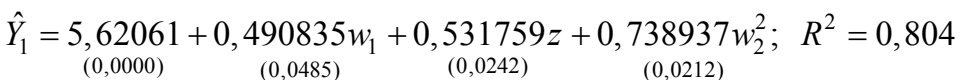

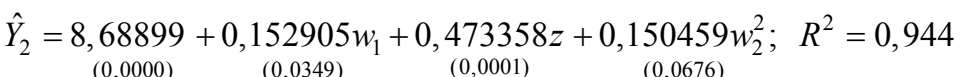

$$
\begin{aligned}
& \hat{Y}_{3}=\underset{(0,0000)}{0,703}-\underset{(0,0005)}{0,047 z}+\underset{(0,0312)}{0,0325} w_{1} w_{2} ; R^{2}=0,865
\end{aligned}
$$

Alternativamente, pode-se calcular valores de preferência diretamente a partir das observações das variáveis de resposta em cada tratamento experimental. Entretanto, a utilização de valores preditos a partir das equações (13) a (15) leva a resultados mais confiáveis, já que toda a variação sistemática observada nas variáveis de resposta foi capturada pelos modelos.

Uma variável de resposta funcional relacionada à textura do biscoito ( $p=4$, designada por textura) foi simulada para ilustrar os cálculos utilizando as eqs. (2) a (5). A simulação foi baseada em testes reais de carga-e-deformação realizados por Manohar \& Rao (1999) em biscoitos. Foram gerados um perfil-alvo e dez perfis alternativos, associados aos tratamentos experimentais na Tabela 2. Os perfis estão apresentados na Figura 3; a curva em negrito corresponde ao perfil-alvo; demais curvas correspondem aos dez tratamentos experimentais na Tabela 2. Os vetores de dados associados aos perfis são apresentados no Apêndice A; o vetor mais curto contém 32 observações ordenadas no tempo e o mais longo, 60 observações (as medições de carga foram interrompidas após uma seqüência de duas leituras zeradas). O perfil-alvo corresponde ao tratamento $t=0$. 


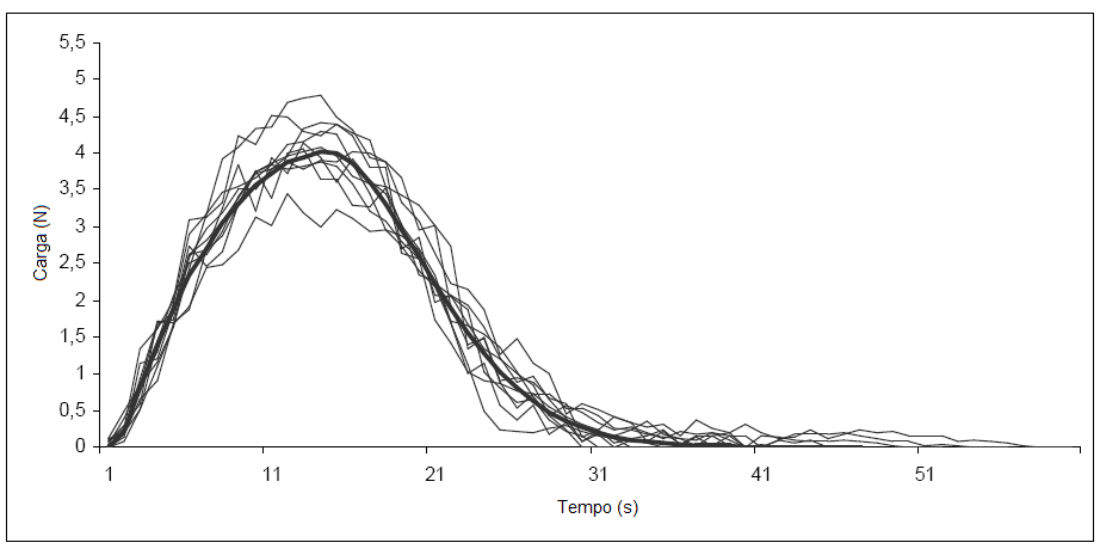

Figura 3 - Observações da variável de resposta funcional $\mathrm{Y}_{4}$ e perfil-alvo (em negrito).

Os valores de distância de Hausdorff associados aos dados no Apêndice A foram calculados programando as eqs. (2) a (5) em Visual Basic para Microsoft Excel (programação disponível mediante solicitação aos autores); esses valores são apresentados na Tabela 3 , sendo designados por $h_{t}^{E}$. Também realizou-se a substituição do operador de máximo na eq. (2) por operadores de mediana e soma; os valores de DH associados a esses operadores são também apresentados na Tabela 3, sendo designados por $h_{m e d, t}^{E}$ e $h_{\text {sum }, t}^{E}$. Os perfis na Figura 3 foram associados a tratamentos experimentais após a determinação de seus valores de $h_{t}^{E}$, tal que o efeito principal $z$ e a interação $w_{2} \times z$ resultassem significativos (nesta ordem) em uma modelagem de regressão usando $h_{t}^{E}$ como variável dependente. As informações na Tabela 3 refletem essa intenção. Os modelos de regressão obtidos são os seguintes (números entre parênteses são valores $p$ de significância associados aos termos dos modelos):

$$
\begin{aligned}
& h_{t}^{E}=\underset{(0,0000)}{0,3395+0,05362} w_{2}-\underset{(0,0001)}{0,15623 z}-0,03653 w_{1} \times z-\underset{(0,000)}{0,0,10113} w_{2} z+\underset{(0,0000)}{0,06875 w_{1}} \times w_{2}
\end{aligned}
$$

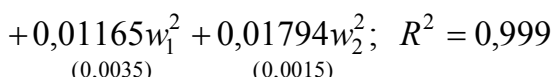

$$
\begin{aligned}
& h_{\text {med, },}^{E}=\underset{(0,0000)}{0,03595-0,00975 z ;} R^{2}=0,576 \\
& h_{\text {sum }, t}^{E}=\underset{(0,0000)}{4,5786}-\underset{(0,0007)}{1,38 z}-\underset{(0,0324)}{0,7203 w_{2}} \times z ; \quad R^{2}=0,850
\end{aligned}
$$

As Figuras 4 e 5 apresentam os tratamentos experimentais com menor e maior valores de $h_{t}^{E}, h_{\text {sum }, t}^{E}$ e $h_{\text {med, } t}^{E}$. As distâncias de Hausdorff calculadas usando $h_{t}^{E}$ e $h_{\text {sum }, t}^{E}$ levaram aos mesmos resultados em termos de melhor e pior tratamentos, os quais são diferentes daqueles obtidos usando $h_{m e d, t}^{E}$. A partir de uma análise visual, todas as medidas identificam satisfatoriamente tratamentos distantes do perfil-alvo, mas usando $h_{\text {med,t }}^{E}$ obtém-se um melhor tratamento mais próximo do perfil-alvo e apresentando menor variabilidade; $h_{\text {med, } t}^{E}$ 
foi, assim, a distância escolhida para finalização desse estudo de caso. Essa escolha parece em desacordo com o desempenho de $h_{\text {med,t }}^{E}$ nos modelo da eq. (17), já que esta medida apresentou o menor coeficiente de determinação dentre as três distâncias modeladas. Cabe ressaltar, entretanto, que a modelagem na eq. (17) associa $h_{\text {med, }}^{E}$ aos fatores experimentais, e não ao perfil-alvo. Uma medida fortemente relacionada aos fatores experimentais (isto é, com $R^{2} \approx 1$ ), não necessariamente resulta em perfis preditos próximos ao perfil-alvo.

Valores de preferência foram calculados para $h_{\text {med, } t}^{E}$ usando suas observações preditas; tais valores são apresentados na Tabela 3 , sendo designados por $d_{4 t}$. Os limites de especificação usados nos cálculos excederam em $5 \%$ os valores extremos observados para as distâncias de Hausdorff, de forma a evitar valores zerados de preferência; são eles: $U_{1}=0,04798 ; L_{1}=0,02489$.

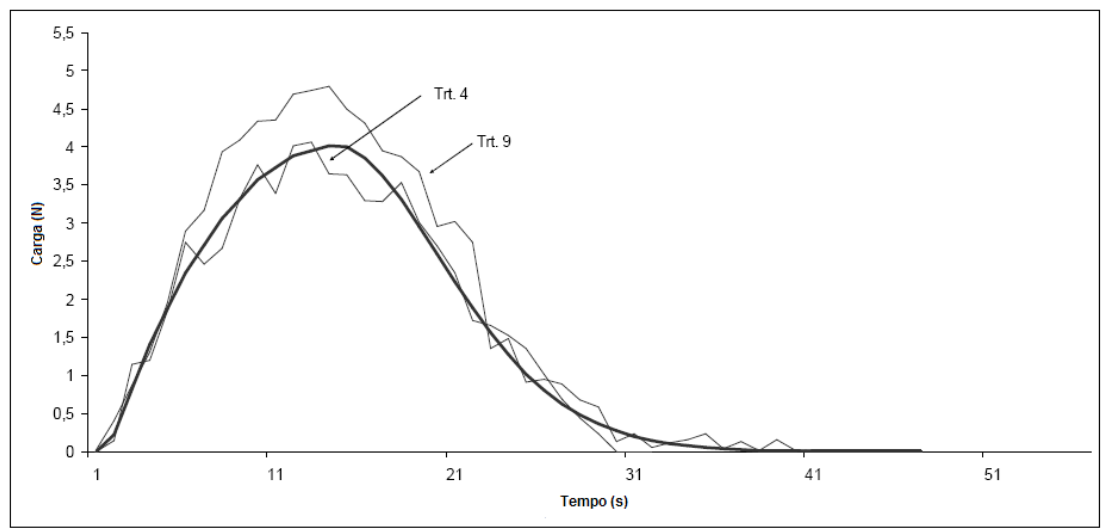

Figura 4 -Melhor (trt. 4) e pior (trt. 9) tratamentos usando $h_{t}^{E}$ e $h_{\text {sum }, t}^{E}$.

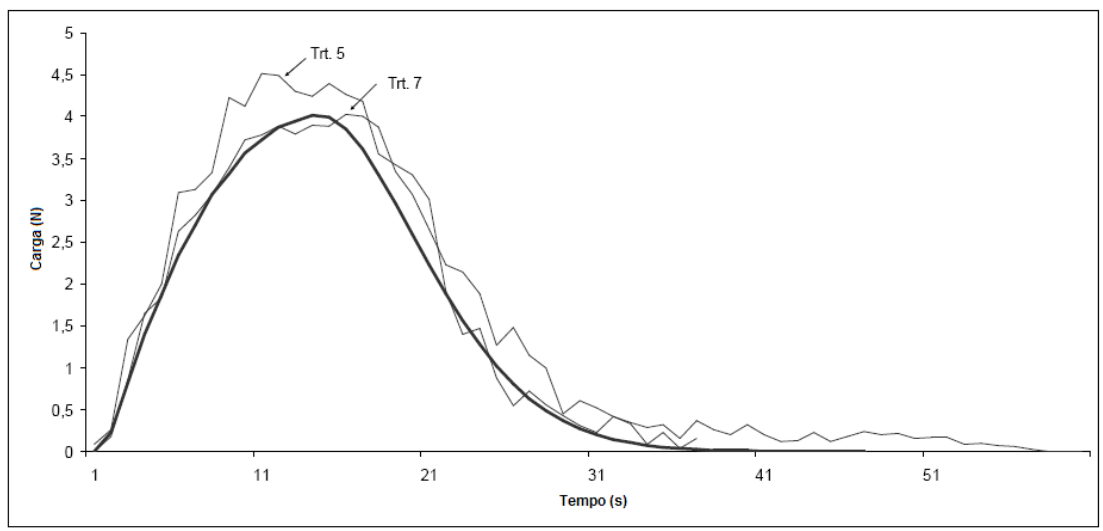

Figura 5 - Melhor (trt. 7) e pior (trt. 5) tratamentos usando $h_{\text {med, },}^{E}$. 
Tabela 3 - Valores de distância de Hausdorff usando diferentes operadores; valores preditos de Hausdorff usando o operador de mediana e seus respectivos valores de preferência.

\begin{tabular}{|c|c|c|c|c|c|c|c|c|}
\hline $\operatorname{Trt}(\boldsymbol{t})$ & $w_{1}$ & $w_{2}$ & $z$ & $h_{t}^{E}$ & $h_{\text {med,t }}^{E}$ & $h_{\text {sum }, t}^{E}$ & $\hat{h}_{\text {med,t }}^{E}$ & $d_{4 t}$ \\
\hline 1 & -1 & -1 & -1 & 0,402 & 0,0450 & 5,109 & 0,046 & 0,0989 \\
\hline 2 & -1 & 1 & -1 & 0,574 & 0,0530 & 6,169 & 0,046 & 0,0989 \\
\hline 3 & 1 & -1 & -1 & 0,339 & 0,0275 & 4,100 & 0,046 & 0,0989 \\
\hline 4 & 1 & 1 & -1 & 0,786 & 0,0450 & 7,233 & 0,046 & 0,0989 \\
\hline 5 & 0 & 0 & -1 & 0,496 & 0,0580 & 7,182 & 0,046 & 0,0989 \\
\hline 6 & $-1,41$ & 0 & 1 & 0,257 & 0,0270 & 3,328 & 0,026 & 0,9433 \\
\hline 7 & 1,41 & 0 & 1 & 0,156 & 0,0195 & 2,779 & 0,026 & 0,9433 \\
\hline 8 & 0 & $-1,41$ & 1 & 0,286 & 0,0240 & 3,745 & 0,026 & 0,9433 \\
\hline 9 & 0 & 1,41 & 1 & 0,152 & 0,0365 & 2,644 & 0,026 & 0,9433 \\
\hline 10 & 0 & 0 & 1 & 0,183 & 0,0240 & 3,497 & 0,026 & 0,9433 \\
\hline
\end{tabular}

Torna-se claro, a partir da análise dos valores de preferência nas Tabelas 1 e 2, que as diferentes variáveis de resposta divergem com relação aos pontos ótimos individuais. As respostas 1 e 2 apresentam o tratamento 7 como o melhor, ao passo que a resposta 3 aponta para os tratamentos 1 e 5. Por outro lado, tratamentos 6 a 10 otimizam a resposta funcional 4 . Assim, a função de preferência combinada ponderada da eq. (12) será usada para determinar o melhor ajuste de compromisso para os fatores experimentais de controle. Dois cenários serão simulados. No cenário 1, somente as respostas 1 a 3, com pesos de importância idênticos, serão incluídas na otimização; no cenário 2 , todas as respostas serão incluídas, o que é possível usando o procedimento aqui proposto para a resposta funcional. Nessa última simulação, serão atribuídos pesos de importância de $20 \%$ para as respostas 1 a 3 , ao passo que a resposta 4 receberá um peso de importância de $40 \%$. Um resumo dos resultados obtidos nas simulações está disponível na Tabela 4, onde $D_{t}^{i}$ é o resultado da eq. (12) para o cenário $i(i=1,2)$.

Tabela 4 - Valores combinados de preferência.

\begin{tabular}{|c|c|c|c|c|c|c|c|c|c|c|}
\hline Trt (t) & $\mathbf{1}$ & $\mathbf{2}$ & $\mathbf{3}$ & $\mathbf{4}$ & $\mathbf{5}$ & $\mathbf{6}$ & $\mathbf{7}$ & $\mathbf{8}$ & $\mathbf{9}$ & $\mathbf{1 0}$ \\
\hline$D_{t}^{1}$ & 0,1931 & 0,2486 & 0,4159 & 0,5073 & 0,2901 & 0,3765 & 0,6566 & 0,6747 & 0,3356 & 0,1351 \\
\hline$D_{t}^{2}$ & 0,1477 & 0,1719 & 0,2341 & 0,2638 & 0,1886 & 0,5436 & 0,7590 & 0,7714 & 0,5074 & 0,2940 \\
\hline
\end{tabular}

A etapa final da otimização consiste na determinação de modelos de regressão relacionando valores combinados de preferência, dados em $D_{t}^{i}(i=1,2)$, a fatores experimentais de controle. Não foi possível obter um modelo de regressão significativo usando $D_{t}^{1}$ como variável dependente; nesse caso, o tratamento experimental que otimiza simultaneamente todas as respostas consideradas no cenário é aquele com maior valor de preferência (ou seja, Trt. 8). Quando $D_{t}^{2}$ é utilizada como variável dependente, obtém-se o seguinte modelo:

$$
D_{t}^{2}=\underset{(0,0000)}{0,38815}+\underset{(0,0034)}{0,18693 z ;} R^{2}=0,678
$$


De acordo com a eq. (19), qualquer tratamento experimental $\operatorname{com} z=+1$ otimizaria as quatro respostas simultaneamente; escolhe-se Trt. 8 como correspondendo à melhor amostra, já que o mesmo apresenta o maior valor calculado de preferência na Tabela 4. Em termos dos ingredientes da formulação $\left(X_{1}, X_{2}\right.$ e $\left.X_{3}\right)$, esse tratamento corresponderia a uma formulação com $X_{1}=7,15 \%, X_{2}=7,15 \%, X_{3}=56 \%$, e espessura ajustada no nível alto. Tais resultados podem ser obtidos usando a transformação inversa apresentada por Cornell (1990, p. 115).

\section{Conclusão}

Neste artigo, propõe-se um método para a inclusão de variáveis de resposta funcionais na otimização de experimentos multi-resposta. O método é baseado no uso da distância de Hausdorff (DH) para transformar respostas funcionais, com observações dadas por vetores de pontos, em respostas ordinárias, com observações dadas por valores individuais. A DH fornece uma medida da distância entre pontos em dois perfis, permitindo incorporar a distância-ao-perfil-alvo como critério de otimização em procedimentos de otimização multiresposta. Ao contrário da distância Euclidiana tradicional, o uso da DH não demanda vetores de entrada de igual dimensão (ou seja, nenhum alinhamento prévio de vetores é demandado). Além disso, para utilizar a $\mathrm{DH}$ não é necessária a modelagem prévia das variáveis de resposta funcionais.

O método proposto é aplicado em um estudo de caso. O estudo relata o desenvolvimento de um produto alimentício para animais, onde ressalta-se a importância de inclusão de variáveis de resposta funcionais na busca pelo melhor produto. Através do estudo de caso, ilustram-se os cálculos demandados para a implementação prática do método proposto.

Ao lidar-se com variáveis de resposta funcionais em procedimentos de $\mathrm{OM}$, a variabilidade da resposta observada pode desempenhar um papel importante. É possível visualizar situações onde dois perfis, quando comparados a um perfil-alvo, apresentem o mesmo valor de DH sendo, entretanto, distintos em termos de suavidade (isto é, variação da curva observada com relação à curva-alvo). Nesses casos, seria natural favorecer perfis próximos ao perfil-alvo que também apresentassem baixa variabilidade. Futuros desenvolvimentos do método aqui proposto devem incluir a suavidade do perfil como um critério de otimização em procedimentos de OM envolvendo variáveis de resposta funcionais.

\section{Agradecimentos}

A pesquisa do Prof. Fogliatto é financiada pelo CNPq, através do processo PQ301433/2005-4. $\mathrm{O}$ autor gostaria de agradecer aos referees anônimos pela atenção dispensada ao artigo e pelas valiosas sugestões feitas visando a sua melhoria.

\section{Referências Bibliográficas}

(1) Antony, J.; Anand, R.B.; Maneesh, M. \& Tiwari, M.K. (2006). Multiple response optimization using Taguchi methodology and neuro-fuzzy based model. Journal of Manufacturing Technology Management, 17(7), 908-925.

(2) Belogay, E.; Cabrelli, C.; Molter, U. \& Shonkwiler, R. (1997). Calculating the Hausdorff distance between curves. Information Processing Letters, 64, 17-22. 
(3) Berni, R. \& Gonnelli, C. (2006). Planning and Optimization of a Numerical Control Machine in a Multiple Response Case. Quality and Reliability Engineering International, 22, 517-526.

(4) Biles, W.E. (1975). A response surface method for experimental optimization of multiresponse processes. Industrial Engineering Chemistry, Process Design and Development, 14, 152-158.

(5) Cardot, H.; Ferraty, F. \& Sarda, P. (1999). Functional linear model. Statistics \& Probability Letters, 45, 11-22.

(6) Castagliola, P. \& Rosa, A.F.P. (2006). Monitoring of Batch Processes with Varying Durations Based on the Hausdorff Distance. International Journal of Reliability, Quality and Safety Engineering, 13(3), 213-236.

(7) Ch'ng, C.K.; Quah, S.H. \& Low, H.C. (2005). Index $C_{p m}^{*}$ in multiresponse optimization. Quality Engineering, 17, 161-175.

(8) Chang, S. \& Shivpuri, R. (1994). A multiple-objective decision-making approach for assessing simultaneous improvement in die life and casting quality in a die-casting process. Quality Engineering, 7, 371-383.

(9) Cheng, C.B.; Cheng, C.J. \& Lee, E.S. (2002). Neuro-fuzzy and genetic algorithm in multiple response optimization. Computers and Mathematics with Applications, 44, 1503-1514.

(10) Cornell, J.A. (1990). Experiments with mixtures: designs, models, and analysis of mixture data. Wiley, New York.

(11) Del Castillo, E.; Montgomery, D.C. \& McCarville, D.R. (1996). Modified desirability functions for multiple response optimization. Journal of Quality Technology, 28, 337-345.

(12) Del Castillo, E. (1996). Multiresponse process optimization via constrained confidence regions. Journal of Quality Technology, 28, 61-70.

(13) Derringer, G. \& Suich, R. (1980). Simultaneous optimization of several response variables. Journal of Quality Technology, 12, 214-219.

(14) Elsayed, E.A. \& Chen, A. (1993). Optimal levels of process parameters for products with multiple characteristics. International Journal of Production Research, 31, 1117-1132.

(15) Ferraty, F. \& Vieu, P. (2006). Nonparametric functional analysis - Theory and practice. Springer, New York.

(16) Fogliatto, F.S.; Albin, S.L. \& Tepper, B.J. (1999). A hierarchical approach to optimizing descriptive analysis multiresponse experiments. Journal of Sensory Studies, 14, 443-465.

(17) Fogliatto, F.S. \& Albin, S.L. (2001). A hierarchical method for evaluating products with quantitative and sensory characteristics. IIE Transactions, 33, 1081-1092.

(18) Fogliatto, F.S. \& Albin, S.L. (2003). An AHP-based procedure for sensory data collection and analysis in quality and reliability applications. Food Quality \& Preference, 14, 375-385.

(19) Fogliatto, F.S. \& Albin, S.L. (2000). Variance of predicted response as an optimization criterion in multiresponse experiments. Quality Engineering, 12, 523-533. 
(20) Fung, C.P. \& Kang, P.C. (2005). Multi-response optimization in friction properties of PBT composites using Taguchi method and principal components analysis. Journal of Materials Processing Technology, 170, 602-610.

(21) Govaerts, B. \& Noël, J. (2005). Analysing the results of a designed experiment when the response is a curve: Methodology and application in metal injection moulding. Quality and Reliability Engineering International, 21, 509-520.

(22) Harrington Jr, E.C. (1965). The desirability function. Industrial Quality Control, 21, 494-498.

(23) Hsieh, K.L. \& Tong, L.I. (2001). Optimization of multiple quality responses involving qualitative and quantitative characteristics in IC manufacturing using neural networks. Computers in Industry, 46, 1-12.

(24) Huttenlocher, D.P. \& Kedem, K. (1990). Computing the minimum Hausdorff distance for point sets under translation. Proc. of 6th Annual ACM Symp. on Comp. Geom. (SCG'90, Berkeley, CA), 340-349.

(25) Huttenlocher, D.P.; Klanderman, G.A. \& Rucklidge, W.J. (1993). Comparing images using the Hausdorff distance. IEEE Transactions on Pattern Analysis and Machine Intelligence, 15, 850-863.

(26) Jeong, M.K.; Lu, J.C. \& Wang, N. (2006). Wavelet-based SPC procedure for complicated functional data. International Journal of Production Research, 44(4), 729-744.

(27) Kang, L. \& Albin, S.L. (2000). On-line monitoring when the process yields a linear profile. Journal of Quality Technology, 32 (4), 418-426.

(28) Khuri, A.I. \& Conlon, M. (1981). Simultaneous optimization of multiple responses represented by polynomial regression functions. Technometrics, 23, 363-375.

(29) Khuri, A.I. \& Cornell, J.A. (1996). Response Surfaces. $2^{\text {nd }}$ ed. Dekker, New York.

(30) Kim, K.; Mahmoud, M.A. \& Woodwall, W.H. (2003). On the monitoring of linear profiles. Journal of Quality Technology, 35, 317-328.

(31) Kim, K.J. \& Lin, D.K.J. (1998). Dual response surface optimization: a fuzzy modeling approach. Journal of Quality Technology, 30, 1-10.

(32) Kim, K.J. \& Lin, D.K.J. (2006). Optimization of multiple responses considering both location and dispersion effects. European Journal of Operational Research, 169, 133-145.

(33) Ko, Y.H.; Kim, K.J. \& Jun, C.H. (2005). A new loss function-based method for multiresponse optimization. Journal of Quality Technology, 37, 50-59.

(34) Kros, J.F. \& Mastrangelo, C.M. (2001). Comparing methods for the multi-response design problem. Quality and Reliability Engineering International, 17, 323-331.

(35) Kulkarni, M.S. \& Mariappan, V. (2003). Multiple response optimization for improved machined surface quality. Journal of Materials Processing Technology, 141, 174-180.

(36) León, N.A. (1996). A pragmatic approach to multiresponse problems using loss functions. Quality Engineering, 9, 213-220.

(37) León, R.V.; Shoemaker, A.C. \& Kacker, R.N. (1987). Performance measures independent of adjustment - an explanation and extension of Taguchi's signal-to-noise ratios. Technometrics, 29, 253-265. 
(38) Li, J.; Ma, C.; Ma, Y.; Li, Y.; Zhou, W. \& Xu, P. (2007). Medium optimization by combination of response surface methodology and desirability function: an application in glutamine production. Appl Microbiol Biotechnol, 74, 563-571.

(39) Lin, J.L. \& Lin, C.L. (2005). The use of grey-fuzzy logic in the optimization of the manufacturing process. Journal of Materials Processing Technology, 160, 9-14.

(40) Lind, E.E.; Goldin, J. \& Hickman, J.B. (1960). Fitting yield and cost response surfaces. Chemical Engineering Progress, 56, 62-68.

(41) Logothetis, N. \& Haigh, A. (1988). Characterizing and optimizing multiresponse processes by the Taguchi method. Quality and Reliability Engineering International, 4, 159-169.

(42) Lu, D. \& Antony, J. (2002). Optimization of multiple responses using a fuzzy-rule based inference system. International Journal of Production Research, 40, 1613-1625.

(43) Manohar, R.S. \& Rao, P.H. (1999). Effect of emulsifiers, fat level and type on the rheological characteristics of biscuit dough and quality of biscuits. Journal of the Science of Food and Agriculture, 79, 1223-1231.

(44) Manteiga, W.G. \& Vieu, P. (2007). Statistics for functional data. Computational Statistics \& Data Analysis, 51, 4788-4792.

(45) Montgomery, D.C.; Keats, J.B.; Perry, L.A.; Thompson, J.R. \& Messina, W.S. (2000). Using statistically designed experiments for process development and improvement. Robotics and Computer Integrated Manufacturing, 16, 55-63.

(46) Mukherjee, I. \& Ray, P.K. (2008). Optimal process design of two-stage multiple responses grinding processes using desirability functions and metaheuristic technique. Applied Soft Computing, 8, 402-421.

(47) Murphy, T.E.; Tsui, K.L. \& Allen, J.K. (2005). A review of robust design methods for multiple responses. Research in Engineering Design, 15, 201-215.

(48) Myers, R.H. \& Carter Jr, W.H. (1973). Response surface techniques for dual response surfaces. Technometrics, 15, 301-317.

(49) Myers, R.H.; Khuri, A.I. \& Vining, G.G. (1992). Response surface alternatives to the Taguchi robust parameter design approach. The American Statistician, 46, 131-139.

(50) Myers, R.H. \& Montgomery, D.C. (2002). Response Surface Methodology: Process and Product Optimization Using Designed Experiments. $2^{\text {nd }}$ Ed. Wiley, New York.

(51) Nair, V.N.; Taam, W. \& Ye, K.Q. (2002). Analysis of functional responses from robust design studies. Journal of Quality Technology, 34, 355-370.

(52) Ortiz, F.; Simpson, J.R.; Pignatielo Jr, J.J. \& Heredia-Langner, A. (2004). A genetic algorithm approach to multiple-response optimization. Journal of Quality Technology, 36, 432-450.

(53) Pasamontes, A. \& Callao, M.P. (2006). Optimization by means of responses surface of an analytical sequence using a sequential injection system. Talanta, 68, 1617-1622.

(54) Pasandideh, S.H.R. \& Niaki, S.T.A. (2006). Multi-response simulation optimization using genetic algorithm within desirability function framework. Applied Mathematics and Computation, 175(1), 366-382.

(55) Pignatiello Jr, J.J. (1993). Strategies for robust multiresponse quality engineering. IIE Transactions, 25, 5-15. 
(56) Plante, R.D. (2001). Process capability: a criterion for optimizing multiple response product and process design. IIE Transactions, 33, 497-509.

(57) Quesada, G.M. \& Del Castillo, E. (2004). A dual-response approach to the multivariate robust parameter design problem. Technometrics, 46, 176-187.

(58) Raiman, L.B. \& Case, K.E. (1992). The development and implementation of multivariate cost of poor quality loss function. IMSE Working Paper; 92-152 (Penn State Univ.).

(59) Ramsay, J.O. \& Silverman, B.W. (2005). Functional data analysis. $2^{\text {nd }}$ Ed. Springer, New York.

(60) Ribeiro, J.L. \& Elsayed, E.A. (1995). A case study on process optimization using the gradient loss function. International Journal of Production Research, 33, 3233-3248.

(61) Ribeiro, J.L.; Fogliatto, F.S. \& ten Caten, C.S. (2001). Minimizing manufacturing and quality costs in multiresponse optimization. Quality Engineering, 13, 559-569.

(62) Shah, H.K.; Montgomery, D.C. \& Carlyle, W.M. (2004). Response surface modeling and optimization in multiresponse experiments using seemingly unrelated regressions. Quality Engineering, 16, 387-397.

(63) Shen, Q. \& Xu, H. (2007). Diagnostics for linear models with functional responses. Technometrics, 49(1), 26-33.

(64) Siegel, I.H. (1942). Index-Number Differences: Geometric Means. Journal of the American Statistical Association, 37(218), 271-274.

(65) Taguchi, G. (1986). Introduction to quality engineering: designing quality into products and processes. Kraus Int. Publ., White Plains (NY).

(66) Tribus, M. \& Szonyl, G. (1989). An alternative view of the Taguchi approach. Quality Progress, 22, 46-52.

(67) Tyan, J.C.; Du, T.C.; Chen, J.C. \& Chang, I.H. (2004). Multiple response optimization in a fully automated FAB: an integrated tool and vehicle dispatching strategy. Computers \& Industrial Engineering, 46, 121-139.

(68) Vining, G.G. (1998). A compromise approach to multiresponse optimization. Journal of Quality Technology, 30, 309-313.

(69) Williams, J.D.; Woodall, W.H. \& Birch, J.B. (2007). Statistical Monitoring of Nonlinear Product and Process Quality Profiles. Quality and Reliability Engineering International, 23, 925-941.

(70) Wu, F.C. (2005). Optimization of correlated multiple quality characteristics using desirability function. Quality Engineering, 17, 119-126.

(71) Wurl, R.C. \& Albin, S.L. (1999). A comparison of mutiresponse optimization: sensitivity to parameter selection. Quality Engineering, 11, 405-416.

(72) Yang, Y. \& Chou, P. (2005). Solving a multiresponse simulation-optimization problem with discrete variables using a multiple-attribute decision-making method. Mathematics and Computers in Simulation, 68, 9-21.

(73) Yum, B. \& Ko, S. (1991). On Parameter Design Optimization Procedures. Quality and Reliability Engineering International, 7, 39-46. 
Apêndice A - Vetores de dados de textura.

\begin{tabular}{|c|c|}
\hline $\operatorname{Trt}(t)$ & Dados \\
\hline 0 & $\begin{array}{l}0 ; 0,226 ; 0,815 ; 1,404 ; 1,877 ; 2,35 ; 2,707 ; 3,063 ; 3,316 ; 3,568 ; 3,723 ; 3,879 ; 3,947 ; \\
4,014 ; 3,997 ; 3,855 ; 3,617 ; 3,311 ; 2,963 ; 2,598 ; 2,233 ; 1,885 ; 1,563 ; 1,275 ; 1,023 ; \\
0,808 ; 0,629 ; 0,482 ; 0,364 ; 0,271 ; 0,199 ; 0,144 ; 0,103 ; 0,073 ; 0,051 ; 0,035 ; 0,024 ; \\
0,016 ; 0,01 ; 0,007 ; 0,004 ; 0,003 ; 0,002 ; 0,001 ; 0,000 ; 0,000\end{array}$ \\
\hline 1 & $\begin{array}{l}0 ; 0,14 ; 1,15 ; 1,2 ; 1,85 ; 2,75 ; 2,463 ; 2,67 ; 3,32 ; 3,77 ; 3,388 ; 4,01 ; 4,07 ; 3,653 \text {; } \\
3,638 ; 3,3 ; 3,28 ; 3,53 ; 3,01 ; 2,7 ; 2,35 ; 1,715 ; 1,66 ; 1,53 ; 1,36 ; 1,02 ; 0,69 ; 0,438 \\
0,23 ; 0,000 ; 0,000\end{array}$ \\
\hline 2 & $\begin{array}{l}0,000 ; 0,183 ; 0,660 ; 1,137 ; 1,520 ; 1,904 ; 2,193 ; 2,481 ; 2,686 ; 2,890 ; 3,016 ; 3,142 ; \\
3,197 ; 3,252 ; 3,238 ; 3,123 ; 2,930 ; 2,682 ; 2,400 ; 2,104 ; 1,809 ; 1,527 ; 1,266 ; 1,033 ; \\
0,829 ; 0,654 ; 0,509 ; 0,390 ; 0,295 ; 0,219 ; 0,161 ; 0,117 ; 0,083 ; 0,059 ; 0,041 ; 0,028 ; \\
0,019 ; 0,013 ; 0,008 ; 0,006 ; 0,004 ; 0,002 ; 0,001 ; 0,001 ; 0,001 ; 0,000 ; 0,000\end{array}$ \\
\hline 3 & $\begin{array}{l}0,000 ; 0,224 ; 0,807 ; 1,390 ; 1,858 ; 2,327 ; 2,680 ; 3,033 ; 3,283 ; 3,532 ; 3,686 ; 3,840 ; \\
3,907 ; 3,974 ; 3,957 ; 3,817 ; 3,581 ; 3,278 ; 2,934 ; 2,572 ; 2,211 ; 1,866 ; 1,548 ; 1,262 ; \\
1,013 ; 0,800 ; 0,622 ; 0,477 ; 0,360 ; 0,268 ; 0,197 ; 0,143 ; 0,102 ; 0,072 ; 0,050 ; 0,034 ; \\
0,023 ; 0,016 ; 0,010 ; 0,007 ; 0,004 ; 0,003 ; 0,002 ; 0,001 ; 0,001 ; 0,000 ; 0,000\end{array}$ \\
\hline 4 & $\begin{array}{l}0,000 ; 0,201 ; 0,725 ; 1,250 ; 1,671 ; 2,092 ; 2,409 ; 2,726 ; 2,951 ; 3,176 ; 3,314 ; 3,452 ; \\
3,512 ; 3,573 ; 3,558 ; 3,431 ; 3,219 ; 2,947 ; 2,637 ; 2,312 ; 1,988 ; 1,678 ; 1,391 ; 1,135 ; \\
0,910 ; 0,719 ; 0,559 ; 0,429 ; 0,324 ; 0,241 ; 0,177 ; 0,128 ; 0,092 ; 0,065 ; 0,045 ; 0,031 ; \\
0,021 ; 0,014 ; 0,009 ; 0,006 ; 0,004 ; 0,003 ; 0,002 ; 0,001 ; 0,001 ; 0,000 ; 0,000\end{array}$ \\
\hline 5 & $\begin{array}{l}0,000 ; 0,203 ; 0,733 ; 1,264 ; 1,689 ; 2,115 ; 2,436 ; 2,757 ; 2,984 ; 3,211 ; 3,351 ; 3,491 ; \\
3,552 ; 3,613 ; 3,598 ; 3,470 ; 3,255 ; 2,980 ; 2,667 ; 2,338 ; 2,010 ; 1,697 ; 1,407 ; 1,147 ; \\
0,921 ; 0,727 ; 0,566 ; 0,434 ; 0,327 ; 0,244 ; 0,179 ; 0,130 ; 0,093 ; 0,065 ; 0,045 ; 0,031 ; \\
0,021 ; 0,014 ; 0,009 ; 0,006 ; 0,004 ; 0,003 ; 0,002 ; 0,001 ; 0,001 ; 0,000 ; 0,000\end{array}$ \\
\hline 6 & $\begin{array}{l}0,000 ; 0,261 ; 0,942 ; 1,624 ; 2,171 ; 2,718 ; 3,130 ; 3,543 ; 3,834 ; 4,126 ; 4,306 ; 4,485 \\
4,564 ; 4,642 ; 4,623 ; 4,458 ; 4,183 ; 3,829 ; 3,427 ; 3,004 ; 2,583 ; 2,180 ; 1,808 ; 1,474 ; \\
1,183 ; 0,934 ; 0,727 ; 0,557 ; 0,421 ; 0,313 ; 0,230 ; 0,167 ; 0,119 ; 0,084 ; 0,058 ; 0,040 ; \\
0,027 ; 0,018 ; 0,012 ; 0,008 ; 0,005 ; 0,003 ; 0,002 ; 0,001 ; 0,001 ; 0,000 ; 0,000\end{array}$ \\
\hline 7 & $\begin{array}{l}0,000 ; 0,236 ; 0,851 ; 1,465 ; 1,959 ; 2,453 ; 2,825 ; 3,197 ; 3,460 ; 3,724 ; 3,886 ; 4,048 ; \\
4,119 ; 4,189 ; 4,172 ; 4,024 ; 3,775 ; 3,455 ; 3,093 ; 2,711 ; 2,331 ; 1,967 ; 1,632 ; 1,331 ; \\
1,068 ; 0,843 ; 0,656 ; 0,503 ; 0,380 ; 0,283 ; 0,208 ; 0,150 ; 0,107 ; 0,076 ; 0,053 ; 0,036 ; \\
0,025 ; 0,016 ; 0,011 ; 0,007 ; 0,005 ; 0,003 ; 0,002 ; 0,001 ; 0,001 ; 0,000 ; 0,000\end{array}$ \\
\hline 8 & $\begin{array}{l}0,000 ; 0,233 ; 0,839 ; 1,445 ; 1,932 ; 2,419 ; 2,787 ; 3,154 ; 3,414 ; 3,673 ; 3,833 ; 3,993 ; \\
4,063 ; 4,133 ; 4,115 ; 3,969 ; 3,724 ; 3,409 ; 3,051 ; 2,674 ; 2,299 ; 1,941 ; 1,610 ; 1,313 ; \\
1,053 ; 0,832 ; 0,647 ; 0,496 ; 0,375 ; 0,279 ; 0,205 ; 0,148 ; 0,106 ; 0,075 ; 0,052 ; 0,036 ; \\
0,024 ; 0,016 ; 0,011 ; 0,007 ; 0,005 ; 0,003 ; 0,002 ; 0,001 ; 0,001 ; 0,000 ; 0,000\end{array}$ \\
\hline 9 & $\begin{array}{l}0,000 ; 0,265 ; 0,954 ; 1,643 ; 2,197 ; 2,751 ; 3,168 ; 3,586 ; 3,881 ; 4,176 ; 4,358 ; 4,540 ; \\
4,619 ; 4,699 ; 4,679 ; 4,513 ; 4,234 ; 3,875 ; 3,469 ; 3,041 ; 2,614 ; 2,206 ; 1,830 ; 1,492 ; \\
1,197 ; 0,946 ; 0,736 ; 0,564 ; 0,426 ; 0,317 ; 0,233 ; 0,169 ; 0,121 ; 0,085 ; 0,059 ; 0,041 ; \\
0,028 ; 0,018 ; 0,012 ; 0,008 ; 0,005 ; 0,003 ; 0,002 ; 0,001 ; 0,001 ; 0,000 ; 0,000\end{array}$ \\
\hline 10 & $\begin{array}{l}0,000 ; 0,249 ; 0,896 ; 1,544 ; 2,065 ; 2,585 ; 2,977 ; 3,370 ; 3,647 ; 3,925 ; 4,096 ; 4,267 ; \\
4,341 ; 4,416 ; 4,397 ; 4,241 ; 3,979 ; 3,642 ; 3,260 ; 2,858 ; 2,457 ; 2,074 ; 1,720 ; 1,402 ; \\
1,125 ; 0,889 ; 0,691 ; 0,530 ; 0,400 ; 0,298 ; 0,219 ; 0,159 ; 0,113 ; 0,080 ; 0,056 ; 0,038 \\
0,026 ; 0,017 ; 0,011 ; 0,008 ; 0,005 ; 0,003 ; 0,002 ; 0,001 ; 0,001 ; 0,000 ; 0,000\end{array}$ \\
\hline
\end{tabular}

